\title{
Morphogens at work
}

How embryos decode morphogen gradients is a fundamental question in developmental biology. Two recent papers address key issues that surround this long-standing problem. The first provides direct evidence that the slope of the morphogen gradient - in this case, that of Decapentaplegic (DPP) - regulates growth during development. The second shows that, contrary to recent predictions, Bicoid (BCD), another morphogen, is sufficient to generate sharp and precisely positioned boundaries of target-gene expression.

Models to explain how morphogen gradients regulate growth and patterning postulate that cells acquire positional values within a gradient, and that proliferation is stimulated

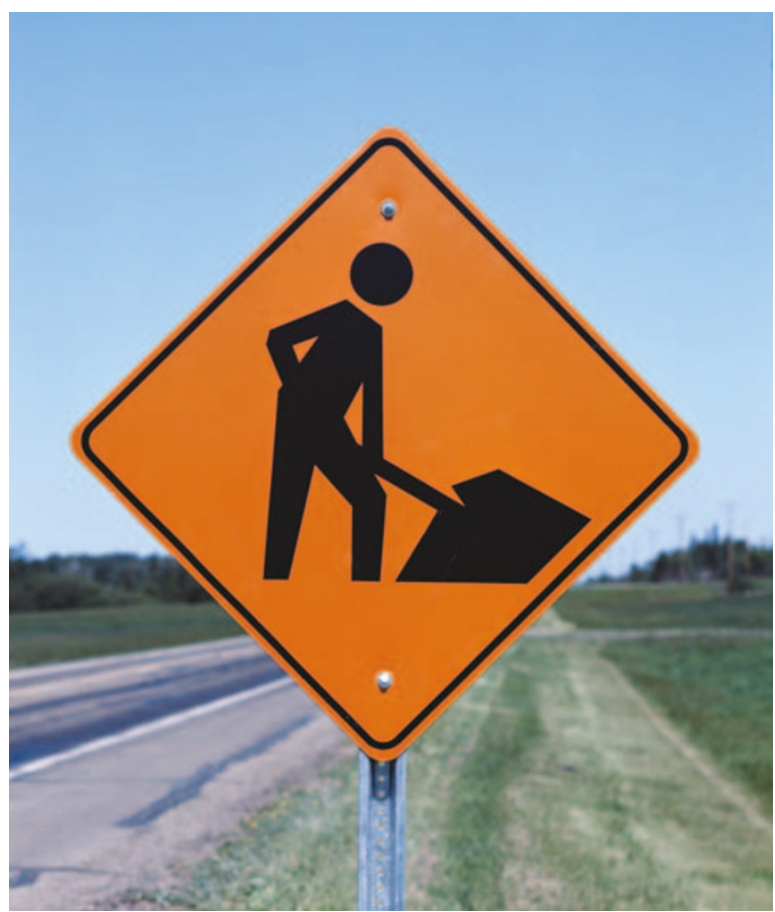

by the detection of differences in these values. However, despite its appeal, this theory has so far lacked experimental support. Rogulja and Irvine reasoned that this could be because previous experiments have focused on longer-term responses, and so decided to study short-term responses to morphogen signals.

Using the fly wing as a model, they devised a method for spatially and temporally controlling gene expression in clones of cells, using a progesterone analogue to activate expression. The morphogen that regulates growth and patterning in the wing is DPP, so the authors made transgenic flies in which a constitutively active form of the DPP receptor, Thick-veins (TKV ${ }^{\mathrm{Q}-\mathrm{D}}$ ), was expressed clonally in the wing. As expected, TKV ${ }^{\mathrm{Q}-\mathrm{D}}$ expression induces the proliferation of cells in a way that depends on their position along the DPP gradient. But, strikingly, TKV ${ }^{\mathrm{Q}-\mathrm{D}}$ expressing cells also induce proliferation in their neighbours, non-cell autonomously. The authors provide several lines of evidence to show that non-autonomous proliferation is induced by the juxtaposition of cells that have different positional values along the DPP gradient. For example, by using different concentrations of the progesterone analogue, they show that non-autonomous proliferation depends on quantitative differences in TKV activation. The authors also made clones of cells in which DPP signalling was inhibited, and showed that non-autonomous proliferation is greatest next to those clones that experience the strongest difference in DPP signal - as expected from the model.

Crauk and Dostatni focused their attention on BCD. In Drosophila melanogaster embryos, the smooth BCD gradient is translated into discrete domains of expression of downstream genes along the anterior-posterior (A-P) axis. Previous reports have suggested that $\mathrm{BCD}$ alone is insufficient to define precise positional information. However, Crauk and Dostatni provide evidence in support of BCD's abilities. They show that a BCD reporter gene, containing binding sites only for BCD in its regulatory region, recapitulates the expression patterns of natural BCD targets. By using deletions in $\mathrm{BCD}$, they define two domains that are important for sharpening the expression of one of BCD's targets (hunchback). Moreover, other transcription factors expressed in a BCD-like maternal gradient also induce sharply defined domains of expression for their targets. This contradicts previous suggestions that an additional molecular filter of a 'noisy' morphogen signal is required to generate precise boundaries.

These two studies show how careful and elegant experimental design can help to resolve long-standing problems in biology. Although classic topics such as morphogen gradients might find themselves less frequently in the limelight these days, here are two examples of why well-established models merit renewed interest.

$$
\text { Magdalena Skipper }
$$

ORIGINAL RESEARCH PAPERS Rogulja, D. 8 Irvine, K. D. Regulation of cell proliferation by a morphogen gradient. Cell 123, 449-461 (2005) | Crauk, O. \& Dostatni, N. Bicoid determines sharp and precise target gene expression in the Drosophila embryo. Curr. Biol. 15, 1888-1898 (2005) 